\title{
PROJECT DESIGN AND IMPLEMENTATION FOR ANKLE WITH MOVING CHARACTERISTICS
}

\author{
Ștefan-Cătălin Popescu, \\ Politehnica University of Bucharest \\ E-mail: popescu.stefancatalin@yahoo.com
}

\begin{abstract}
Today's ankle prostheses tend to improve the walking experience of people with an ankle. Several types of engines are developed to meet the torque and power requirements of an ankle-leg complex with low energy consumption. The ankle prostheses on the market have shown the advantage of using this elastically acting device, being able to deliver maximum ankles $( \pm 120$ $\mathrm{Nm}$ ) and power ( \pm 250 watts) with a $60 \mathrm{~W}$ motor. In this article, the author describes an ankle prosthesis that uses two locking mechanisms as a method of catching energy while walking. The designed ankle prosthesis is powered by a Maxon EC 45 engine. This article aims to mechanically design a device and verify its principle of operation.
\end{abstract}

Keywords: Ankle prosthesis, Bionic legs, Prosthetic.

\section{Introduction}

To replace the missing member, many leg prostheses were designed to restore core functional mobility [1].

The selection of the prosthesis is based on the difficulties of the amputee. The higher the level of amputation of the lower limbs, the greater the amputated person's effort [2].

The design of ankle prostheses has become an important activity of interest for robotics due to technological advances in computer-aided design (CAD) and mechatronics.

\section{Ankle Prosthesis Design Elements}

The drive system used to make this ankle prosthesis consists of two springs that lie behind a locking mechanism.

While driving, this mechanism uses the accumulated energy to help the person with disabilities.

The mechanism has the advantage of accumulating energy and releasing it when necessary. By using two locking mechanisms, the engine can operate for as long as possible.

This new type of drive presents the utility of the mechanism.

The locking mechanisms help to accumulate the energy of the device.

The mechanism does not use cables.

Figure 1 shows the pieces that make up the designed ankle prosthesis.

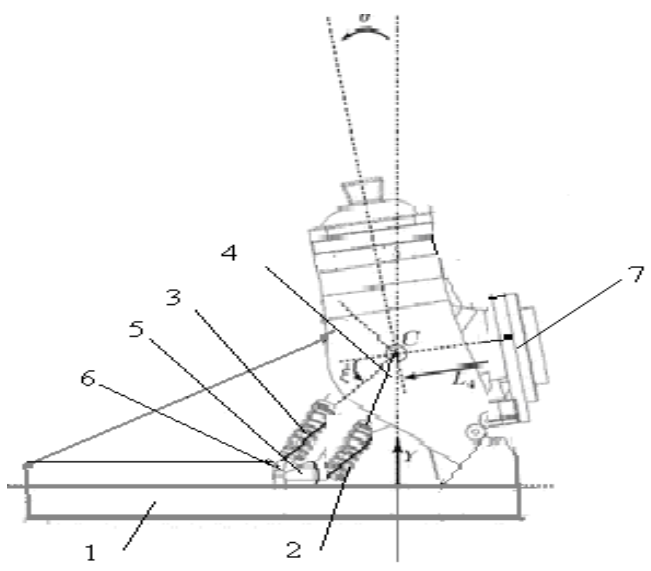

Figure 1: Proposed ankle prosthesis:

1 - ankle, 2 - $\operatorname{arc} 1,3$ - arc 2, 4 - locking mechanism,

5 - cursor, 6 - ankle wrist, 7 - pretensioner, 8 - motor

Figure 2 shows the joint between the prosthesis foot and the ankle prosthesis foot, and Figure 3 shows the engine attached to the ankle prosthesis joint.

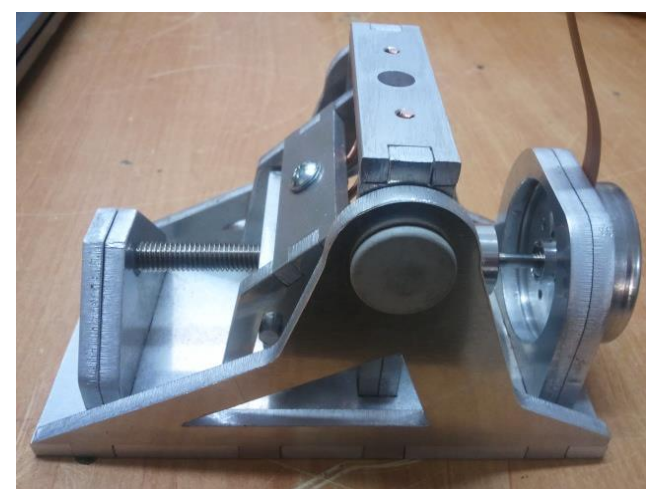

Figure 2: Joint between the ankle foot And the ankle prosthesis foot 


\section{Ankle Operation}

\subsection{The energy concept}

The main objective is to pursue the principle of optimal power distribution in a prosthetic foot, ie the accumulation of as much energy as possible and the use of an electric motor with low energy consumption.

Figure 3 shows the engine attached to the ankle prosthesis joint.

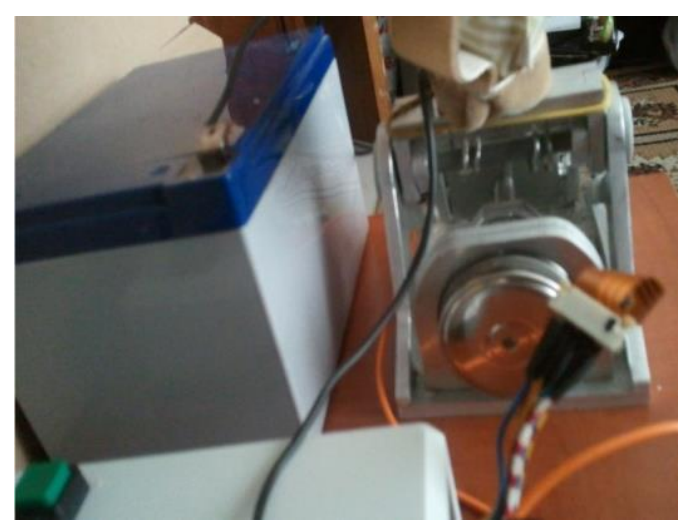

Figure 3: The engine attached to the ankle prosthesis
By writing down the actual mass, the Fe power of the engines and the damping, are defined as follows:

$$
\begin{aligned}
& M_{e}=I_{m} R^{2} \\
& F_{e}=T_{m} R \\
& b_{e}=b_{m} R
\end{aligned}
$$

The engine is modeled as a torque source $\mathrm{Tm}$ with a rotary internal inertia $\mathrm{Im}$, acting the two arcs ks through a transmission $\mathrm{R}$.

The state variables $\mathrm{x}$ and $\theta \mathrm{m}$ measure the linear and rotational displacements of the engine, respectively, where:

$$
x=\frac{\theta_{m}}{R}
$$

The engine that was selected is the Maxon EC 45 engine.

Figure 4 shows the operating region of the engine.

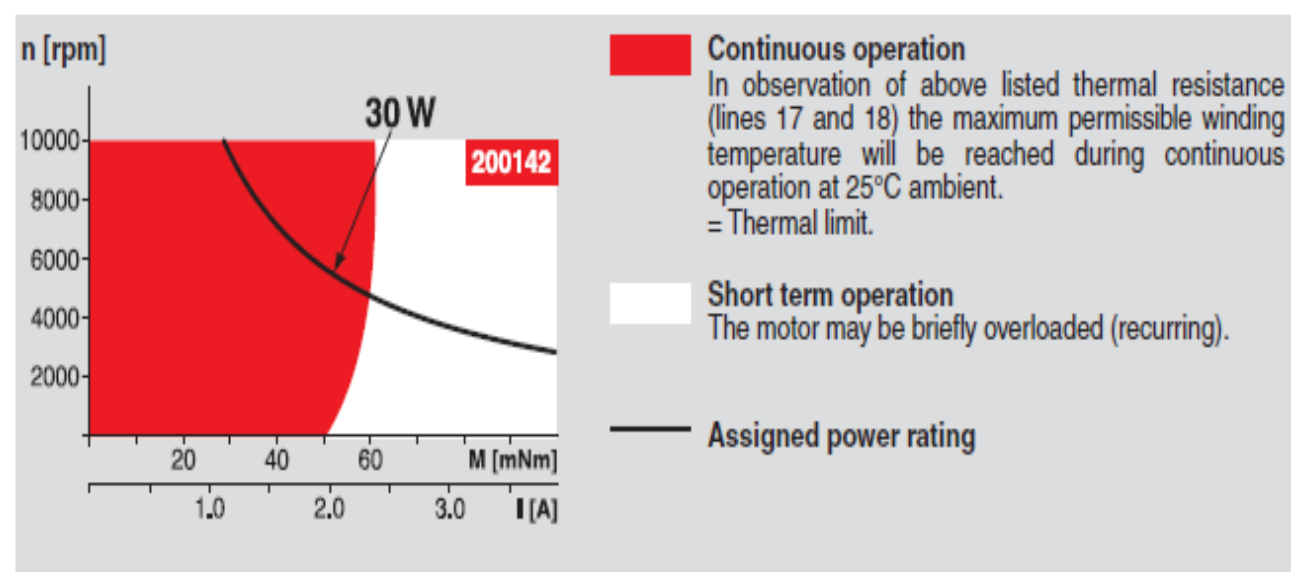

Figure 4: Operating area of a brushless motor (Maxon EC 45) on: www.maxonmotor.com/maxon/view/product/200142 [3]

\subsection{Engine Control}

The engine has three Hall sensors that are located at $120^{\circ}$ from each other and indicate the rotor position. These three sensors divide the revolution into six different phases. The motor has 3 windings that are arranged so as to create six conduction phases that overlap with the sensor phases.

The applied stresses are block-shaped, as do the currents.

The engine runs at constant speeds in simulations. The voltage can be calculated using the speed constant given in the engine data sheet.
The following points have been noted at this stage:

- the motor and the elastic coupling must be placed in line on the foot of the prosthesis;

- the engine has a diameter of $45 \mathrm{~mm}$ and the positioning of the springs near the engine would occupy the space available from the width of the ankle. This would not lead to a compact design;

- must allow for a movement of inversion and eversion that would bring the behavior of the prosthesis closer to that of a healthy ankle;

- the locking mechanisms must be placed on the threaded shaft. An overview of this principle can be found in Figure 5 [3]. 


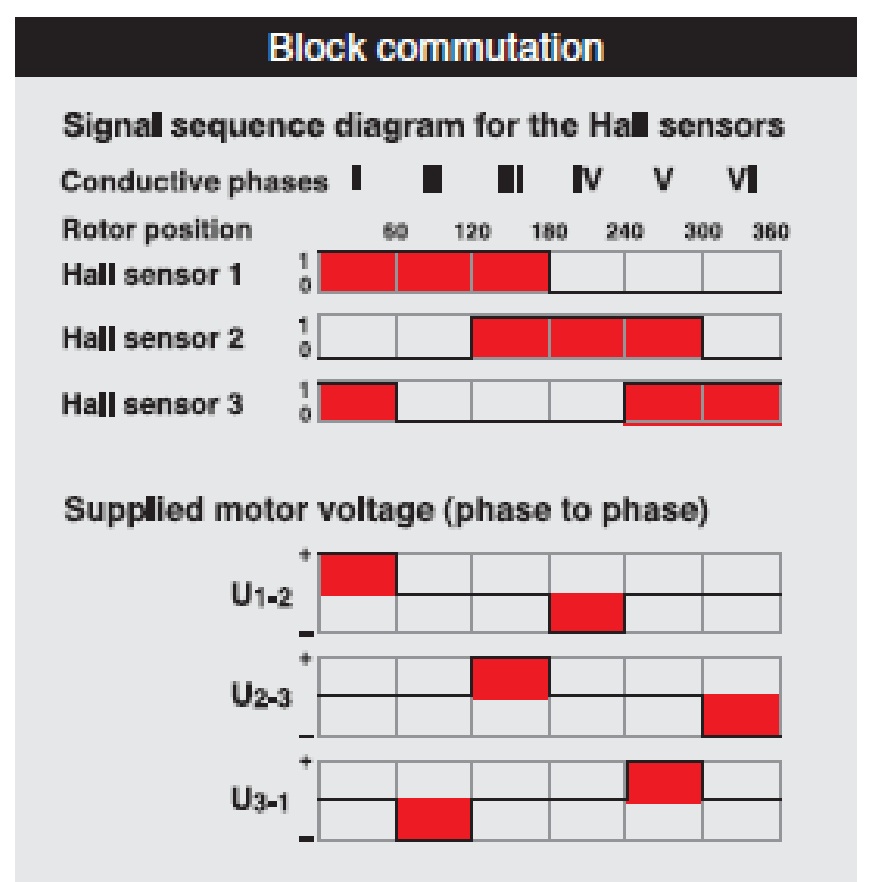

Figure 5: Hall sensor signals and motor voltage supplied for a brushless DC motor. Image adapted from Maxons Technology - Datasheet on: www.maxonmotor.com/maxon/view/product/200142 [3]

\section{Results of Modeling with INVENTOR}

The ankle prosthesis, powered by an engine, must be robust to withstand shocks.
Ankle prosthesis design [4] was a challenge in the area of the ankle joint to effectively reduce shocks and protect the engine from damage.

Figure $6 \mathrm{a}$ and $\mathrm{b}$ show the design of the side, the ankle prosthesis, and the ankle joint.

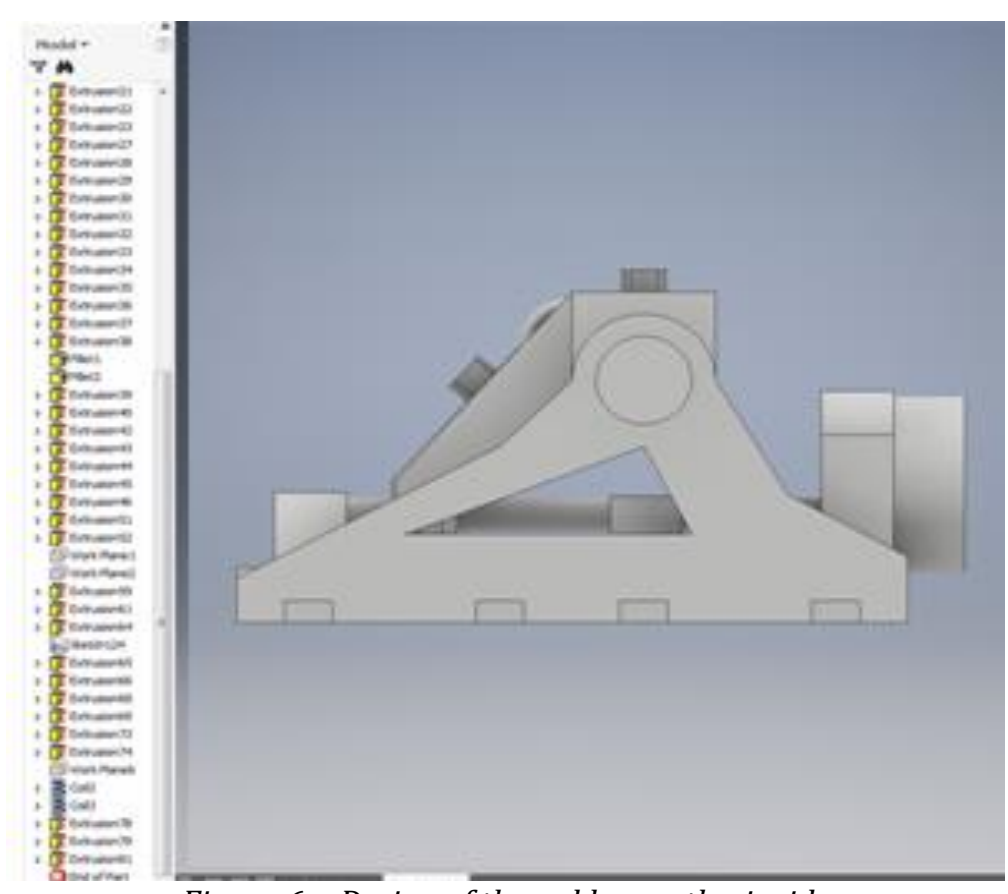

Figura 6a: Design of the ankle prosthesis side 


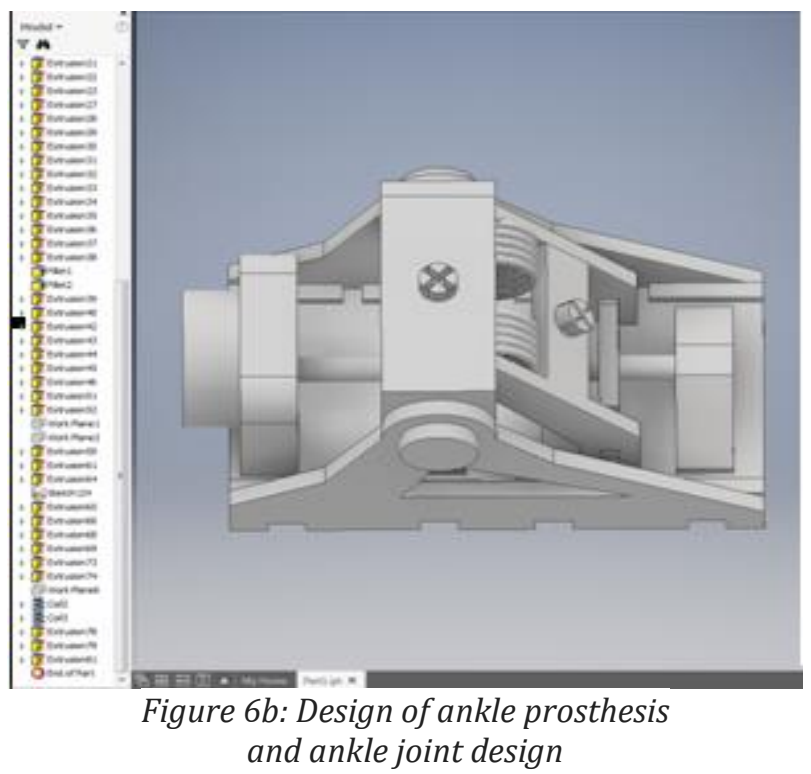

I studied the factors acting on the foot of the prosthesis in the tension zones on the axes XX, YY, Figure 7 and Figure 8.

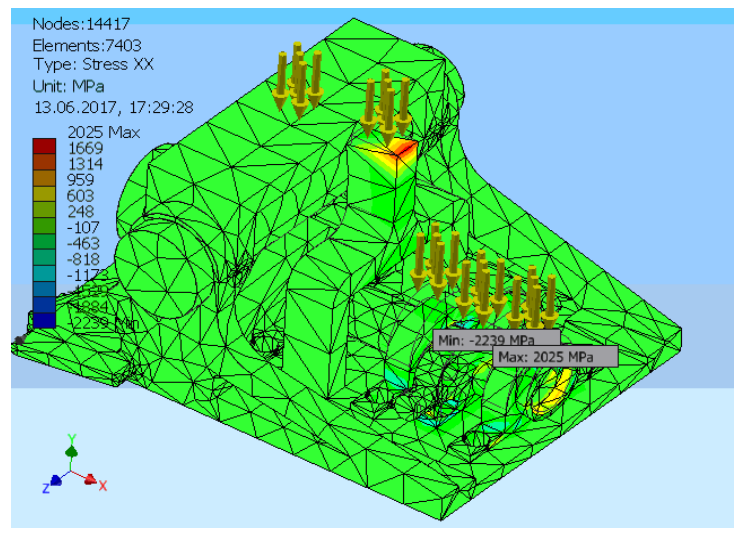

Figure 7: Tensions XX

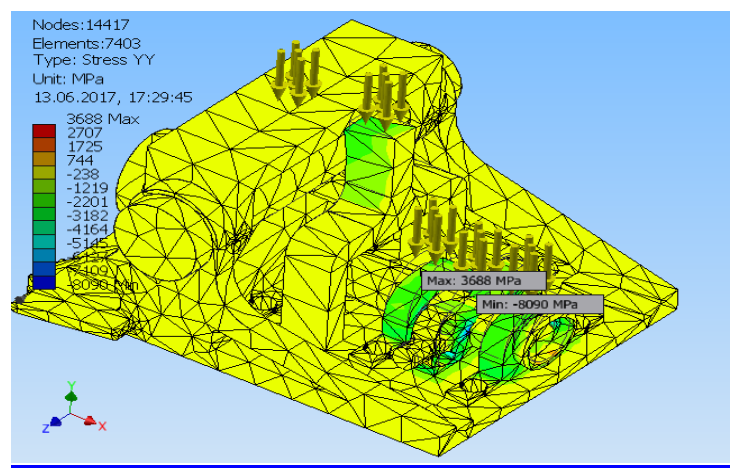

Figure 8: Tensions $Y Y$

\section{Principle of Operation}

The projected ankle prosthesis, Figure 9, consists of three bodies that rotate around a common axis, the ankle axis point $\mathrm{C}$, i.e. the foot and two lever arms, represented as the lever arms 1 and 2 .
The engine assembly is mounted on the foot. The system comprises two sets of springs, placed between the foot and the slider of a sliding mechanism, point A'A ", used to accumulate and release motion energy.

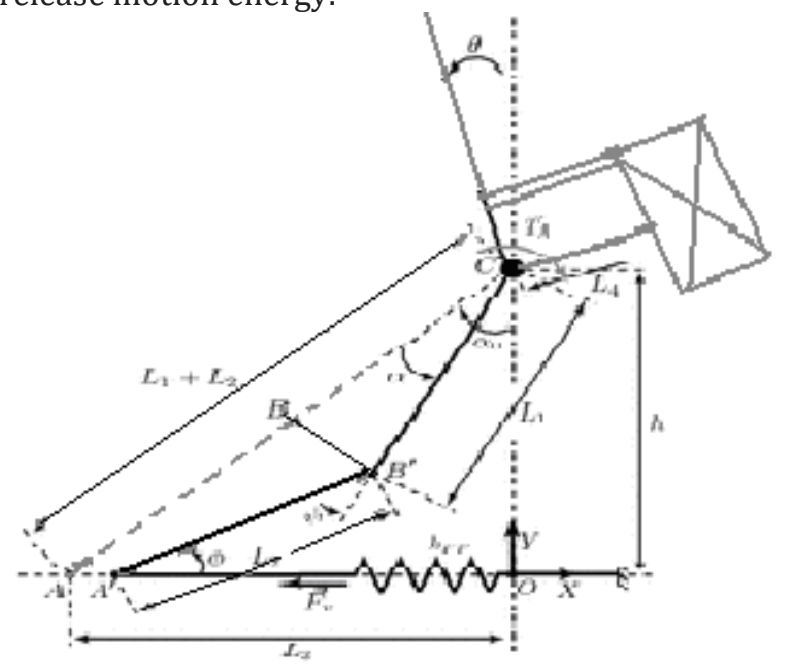

Figure 9: Function of the ankle prosthesis

The lever 1 is the lever of the latter. The link bar is placed between the lever, point $\mathrm{B}^{\prime} \mathrm{B}$ " and slider, point A'A ". The foot and engine forces are transmitted to the foot via this sliding mechanism. This ankle prosthesis was chosen because it improves the system's reliability. The arch is seated between the bonnet assembly and a fixed point (D) located on the lever arm 2. The energy of motion in the PF arc is stored, and the low-power actuator presses on the PO spring without affecting the ankle joint.

The energy accumulated in the PO arc is used and added to the energy stored in the PF arc assembly when a pulse is needed. This energy addition is inserted into the joint of the ankle prosthesis and thus ensures the desired forces and moments of propulsion during walking. 
As mentioned, two locking mechanisms are used. The locking mechanism 1 provides the connection between the two arms. This locking mechanism is used to maximize the added motion energy during the average period.

The second advantage of this locking mechanism is a better movement resembling the characteristics of human walking, ensuring a change in the resting position of the PF spring after the foot is stabilized and the ankle enters the dorsiflexion phase.

The locking mechanism 2 allows a rigid link between the leg and the lever arm when the energy is sent into the system by the electrical unit.

Figure 10 shows the mounting of the locking mechanism, and in Figure 11 the springs are mounted.

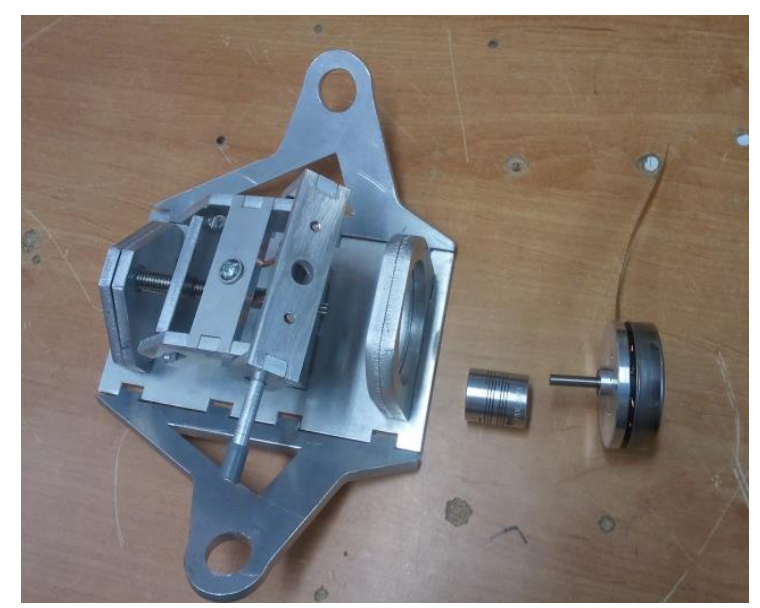

Figure 10: Mounting the locking mechanism

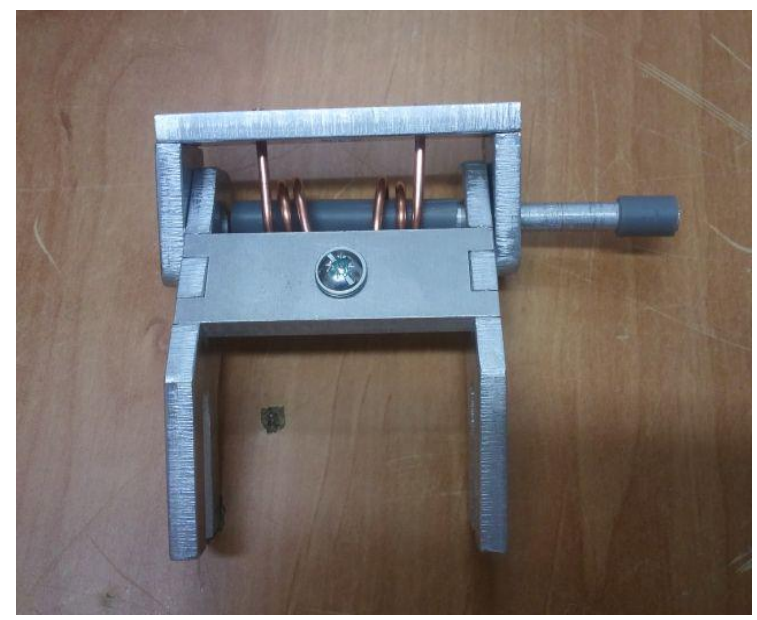

Figure 11: Mouning the springs

Once the foot begins to move in the direction of $\theta$ $=-5^{\circ}$ to $+10^{\circ}$, the reset mechanism is activated by fixing the lever arm 1 to the lever arm 2 .

This is fixed to the foot thanks to the second locking mechanism. The two previously stretched voltage springs in phase 1 are fixed.

They no longer offer any torso for the ankle joint. While they are still elongated, their action is removed from the system.
These springs will remain elongated until the mechanism is switched off at the beginning of the oscillation phase. The energy accumulated in these springs will help reset the ankle.

The lever tracks foot movement. The upward movement of the foot is made by stretching the springs. Torque is generated at the ankle joint by actuating the sliding lever mechanism. Due to the use of the locking mechanism 1 , the motion energy is accumulated in the arches as soon as the ankle carries the dorsiflexion movement of approximately $-5^{\circ}$, depending on the mode of walking of the user. Additional energy storage is between 5 and $10 \mathrm{~J}$.

Figure 12 shows the attachment of the engine. Figure 13 shows the manufactured ankle prosthesis.

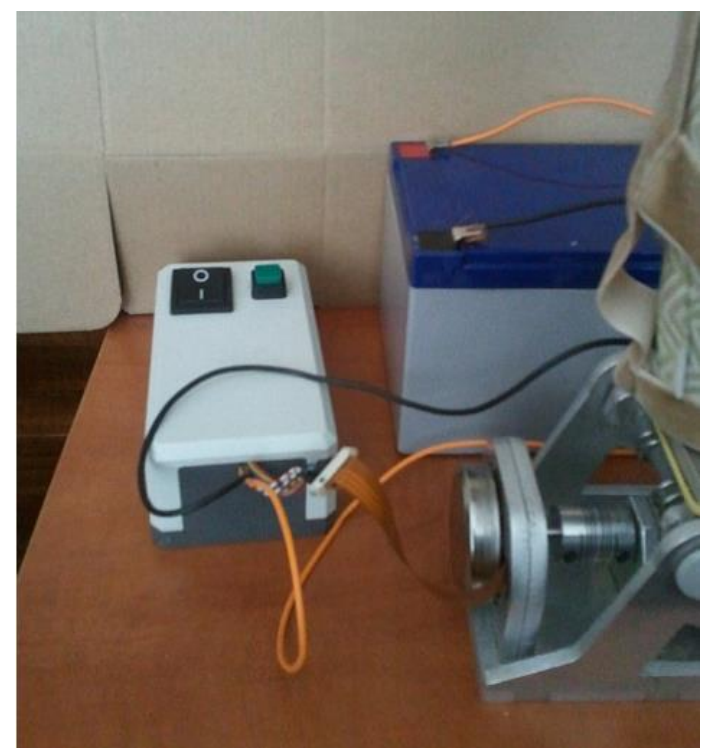

Figure 12: Engine attachment

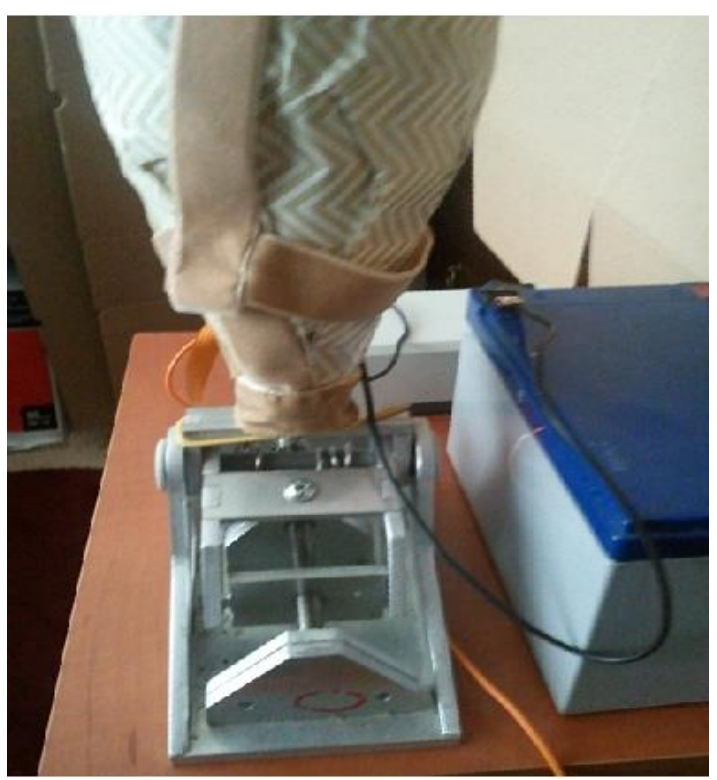

Figure 13: Ankle prosthesis made 


\section{Conclusions}

The author proposed in this article a new model of an energy-efficient ankle prosthesis that imitates ankle behavior by combining elastic spring action with an additional locking mechanism.

The purpose of this study is to manufacture an ankle prosthesis that gathers energy during the movement from the controlled dorsiflexion phase.

The energy is accumulated in two springs, and there is also an energy produced by the low-power electric motor. This energy is released to act on the patient's foot. The mechanical properties of the ankle prosthesis showed positive results.

The disadvantage of this system is its weight of about $3 \mathrm{~kg}$, which is acceptable for a prosthetic leg. The ankle prosthesis can be added with a tracking system and the additional locking system can be improved to provide automatic adjustment at different speeds and gait.

\section{References}

[1] Carroll, K. and Edelstein, J. E., Prosthetics and Patient Management: A Comprehensive Clinical Approach, SLACK Incorporated, 2006. Google Scholar

[2] Bowker, J., Goldberg, B., and Poonekar, P., Atlas of Limb Prosthetics: Surgical, Prosthetic, and Rehabilitation Principles, American Academy of Orthopaedic Surgeons, 1992. Google Scholar

[3] www.maxonmotor.com/maxon/view/product/2 $\underline{00142}$

[4] INVENTOR project: Available at http://www.autodesk.com/products/inventor/o verview/last visited in 28.10.2018.

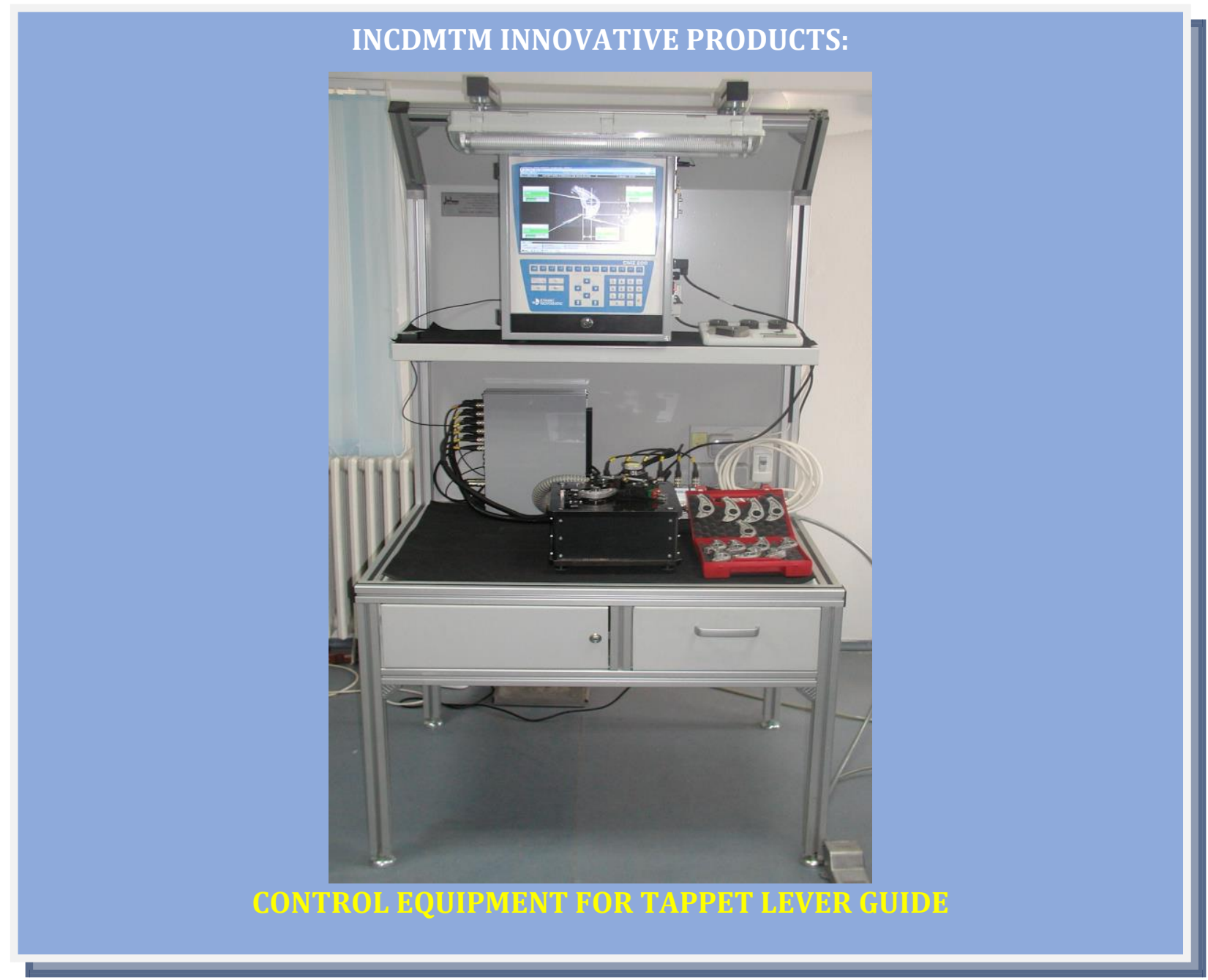

